\title{
Cambio lingüístico y expansión: análisis de "todo" con función citativa en español*
}

\author{
Linguistic change and expansion: An analysis of "todo" with a \\ quotative function in Spanish
}

\section{Eduardo Dominiccini**}

\begin{abstract}
RESUMEN
La tradición gramatical y la semántica formal han relacionado

el determinante "todo" con los significados de cuantificación, modificación adverbial y modificación atributiva. El presente trabajo analiza el uso de este elemento en contextos en los que presenta un significado adicional a estos. En particular, se demuestra que al igual que su contraparte en lenguas como el inglés, todo está registrado en usos coloquiales para introducir formas canónicas y alternativas de discurso directo. Los datos presentados - extraídos de la red social Twitter y recolectados con el programa R- sugieren que "todo" ha desarrollado una función pragmática y ha entrado a formar parte del sistema de citativos en español. El análisis de las estructuras y los usos de "todo" con función citativa se lleva a cabo en términos de la noción de expansión y se relaciona con la discusión acerca de si este tipo de significados son el producto de un proceso de gramaticalización entendida como la puesta en marcha de un mecanismo de analogía.
\end{abstract}

Palabras clave: discurso directo, citativos, gramaticalización, expansión, analogía.

\footnotetext{
* Una versión preliminar de este trabajo fue presentada como actividad final del seminario "Aspectos del cambio lingüístico" (LET4509), que forma parte del programa de Doctorado en Lingüística de la Pontificia Universidad Católica de Chile. Agradezco a la doctora y profesora Verónica Orqueda por su orientación y valiosos comentarios respecto del trabajo. También agradezco los comentarios y preguntas de colegas y participantes del II Coloquio de Lingüistica Histórica (PUC, Santiago), evento llevado a cabo en noviembre de 2018 y en el que presenté un avance de esta investigación. Finalmente, agradezco a los pares evaluadores de la revista por sus comentarios y sugerencias en cuanto a la forma y al contenido de este artículo.

** Candidato a Doctor en Lingüística, Pontificia Universidad Católica de Chile, Santiago, Chile.ledominiccini@uc.cl
} 


\begin{abstract}
Grammatical tradition and formal semantics have associated determiner "todo" ('all') with meanings of quantification, adverbial modification, and attributive modification. This article analyzes this element's use in contexts in which it shows an additional meaning to those mentioned. In particular, this study shows that, like its counterpart in languages such as English, Spanish "todo" can be used to introduce canonical and alternative forms of direct speech. The data presented -extracted from the social network Twitter and collected with the R software- suggest that "todo" has developed a pragmatic function, and has become part of the Spanish's quotative system. The analysis of structures and the use of quotative todo is carried out within the notion of expansion. We discuss whether this type of meaning is a product of a grammaticalization process understood as implementing an analogy mechanism.
\end{abstract}

Keywords: Direct speech, Quotatives, Grammaticalization, Expansion, Analogy. 


\section{Introducción}

En español, además de las formas tradicionales de introducir explícitamente citaciones propias o ajenas o "discurso directo" (en adelante, DD), como el caso de (1), existen formas que se podrían considerar como no canónicas, pero cuya función citativa es la misma (Gallucci, 2017; San Martín y Guerrero, 2013). Algunas de estas estructuras se ejemplifican en (2):

(1) Sujeto + V citativo + "cita"

a. María dijo: "A mí me gustan las películas de terror"

(2) Sujeto $+\emptyset+$ "cita"

a. Y ella, "Ah no, mi'jo" (Cameron, 1998)

Sujeto + (SER) como + "cita"

b. pero sabes era como "ooohhhh Toto eres el mejor" (Jørgensen, 2012)

Sujeto + (SER) todo + "cita"

c. Él todo tímido y ella toda, “;Un placer! ¡Chau!” (Twitter)

El caso de (2a) remite a una variedad de formas que introducen DD sin la presencia de un verbo de habla o de otros elementos, fenómeno que se conoce como "discurso directo libre", "discurso sin marco", "citación cero o nula" o "citación elíptica" (Cameron, 1998; Palacios, 2013)1. La estructura en (2b) presenta el "como" citativo, que se ha analizado en términos generales de manera análoga con la forma del inglés $B E$ like (Jørgensen, 2012; Jørgensen y Stenström, 2009). El caso de (2c), que se aborda en este estudio, equivaldría a lo que se ha registrado en inglés con (be) all. Este uso de all como un citativo se empieza a registrar solo hasta finales del siglo XX (Buchstaller \& Traugott, 2006). La presente investigación exploró esta tendencia para el caso del español todo. Específicamente, el objetivo fue establecer qué características lingüísticas conectaban este uso citativo con las otras funciones de todo en el marco del contexto teórico de los estudios referidos a la gramaticalización.

Cuando el sujeto es realizado por un pronombre personal, esta estructura corresponde a lo que San Martín y Guerrero (2013) denominan "discurso directo con pronombre personal", pero como bien lo señala un evaluador, las citas tipo DD + SN son distintas de las que se dan como discurso libre o freestanding quotation. 
La motivación que impulsó este estudio responde a un vacío en la bibliografía. A pesar de que se reconoce que inglés y español comparten las funciones citativas en elementos como BE/SER + "cita" o like/como + "cita", a la fecha no existen fuentes respecto de la función citativa de todo, cuyas contrapartes en inglés y en otras lenguas germánicas sí han sido estudiadas (Buchstaller, 2014). En efecto, los estudios de gramaticalización a la fecha no han tratado el tema de los nuevos ${ }^{2}$ elementos citativos del español con suficiente profundidad. En Garachana (2015), se menciona brevemente que la construcción $i r+y$ unida al verbo decir ha resultado en su especialización como citativo dentro de un proceso de gramaticalización. Etxepare (2008) y Demonte y Fernández (2013) describen características sintácticas del que citativo, pero no lo tratan desde una perspectiva diacrónica. Por otra parte, Jørgensen (2012) y Panussis y San Martín (2017) mencionan (y no analizan) el uso citativo de como en el español de Madrid y Santiago, pero su estudio tiene un énfasis sociolingüístico más que histórico.

En este contexto, el presente trabajo se centra en datos concretos y en una discusión acerca de las funciones semánticas y pragmáticas de todo. De la literatura previa se retoman dos ideas centrales: las funciones específicas de todo en español y los procesos de cambio que podrían dar cuenta de por qué esta unidad ha devenido en un elemento con función citativa.

El trabajo está organizado de la siguiente manera. En la sección 2 se presentan los antecedentes conceptuales que sirven de base para la discusión respecto de la unidad lingüística central del análisis. La sección 3 describe las características principales del citativo inglés all y explora la posibilidad de una versión citativa en español. La sección 4 aborda la metodología usada en este estudio, incluyendo el procedimiento de obtención de los datos y los aspectos éticos relacionados con su tratamiento. En la sección 5 se presenta un resumen de los resultados de las búsquedas obtenidas en la plataforma Twitter. La sección 6 ofrece una discusión acerca de los datos, que demuestran

2 Con "nuevos" se sigue la tendencia de la bibliografía al hablar de innovaciones en el sistema de los citativos en varias lenguas, en tanto que no hacen parte de los estudios tradicionales referentes a marcadores discursivos. En efecto, la mayoría de estos usos tiene varias décadas, lo cual no es significativo comparado con los siglos de historia de otros citativos. 
la existencia de la función citativa en cuestión, además de un análisis de varios aspectos referidos a los procesos de cambio lingüístico involucrados en los distintos usos de todo en español. Por último, la sección 7 presenta algunas conclusiones y sugerencias de investigación ulterior.

\section{Antecedentes teóricos: las funciones semánticas de todo}

El todo en español proviene del adjetivo latino totus, cuyos orígenes son inciertos y discutibles (de Vaan, 2008). Desde la perspectiva de la gramática tradicional, los estudios acerca del paradigma todo/toda, todos/todas han arrojado resultados variados, debido en parte a la distribución heterogénea de estos elementos y sus propiedades relacionadas con las de adjetivos, pronombres y adverbios (Leonetti, 2007; Real Academia Española, RAE y Asociación de Academias de la Lengua Española, ASALE, 2009). De manera general, se acepta que todo puede corresponder a distintos tipos de categorías, pero su relación conceptual con una idea de cantidad - que es la base del análisis semántico-, tiende a estar presente en la mayoría de los usos conocidos. A continuación, se describe brevemente el conjunto de funciones que desempeña este elemento.

En primer lugar, todo se distingue por ser un cuantificador, de modo que podemos señalar su función cuantificacional $\left(\operatorname{todo}_{\mathrm{FC}}\right)$. Desde la perspectiva de la semántica formal, se han desarrollado marcos de análisis más apropiados para dar cuenta de las propiedades cuantificacionales de este tipo de unidades (Gutiérrez-Rexach, 2016). Según dicha perspectiva, expresiones como todos y algunos — que forman parte de la clase de los determinantes-, se analizan como cuantificadores, es decir, unidades que especifican algún tipo de información acerca de la cantidad. La primera es un cuantificador universal o fuerte (se aplica a la totalidad de elementos de un conjunto), en contraste con la segunda, de valor existencial o débil (determina conjuntos cuantificados no en su totalidad). Respecto de su distribución, Leonetti (2007) señala que el determinante todo $\mathrm{FC}_{\mathrm{FC}}$ presenta características diferentes según precede a nombres escuetos o a sintagmas nominales (en adelante SSNN) definidos. Esto se puede ver en los ejemplos mencionados por este autor:

(3) Toda precaución es poca. 
(4) Todos los concursantes han contestado al mismo tiempo.

En (3), el dominio de cuantificación no está contextualmente restringido, lo que significa que la interpretación del nominal es inespecífica, sin referencia a una entidad en particular. En contraste con (3), la inclusión de un determinante en el sintagma en (4) permite la interpretación referencial que restringe contextualmente el dominio del cuantificador. En general, los SSNN que suceden a todo $\mathrm{F}_{\mathrm{FC}}$ pueden ser singulares o plurales y tienen concordancia de género y número con el cuantificador (RAE/ASALE, 2009).

Como cuantificador, todo $\mathrm{FC}_{\mathrm{FC}}$ alude al conjunto total de entidades de la clase designada por el nombre al que determina. Cuando el nombre está ausente, la contribución básica de todo es la que corresponde a su función pronominal (todo ${ }_{\mathrm{FPRO}}$ ). Este uso es común en el sentido de 'todo el mundo' o 'todas las personas' en expresiones como el proverbio $\mathrm{Del}$ árbol caído, todos hacen leña. En algunos casos, todo funciona como pronombre por efecto de la elipsis de un sustantivo que se recupera contextualmente de manera anafórica, como en Las casas eran viejas, pero todas [las casas] estaban en buen estado (RAE/ASALE, 2009).

Existen otros contextos en los que todo presenta la capacidad de modificar adjetivos, como se ejemplifica en (5):

(5) a. Jajajaja yo estoy igual, voy a terminar toda contracturada! (Twitter)

b. Yo toda enamorada y a punto de un colapso mental después de escuchar Fake Love (Twitter)

El uso de toda en (5a) y (5b) se ha equiparado con el uso de expresiones adverbiales como por completo o completamente (RAE/ASALE, 2009), de modo que el sintagma "toda contracturada" sería interpretado como 'completamente contracturada', mientras que "toda enamorada" equivaldría a 'completamente enamorada'. En estos casos, toda parece tener valores que, aunque podrían estar relacionados de algún modo con una noción abstracta de cantidad (al igual que la forma emparentada totalmente), no corresponden con los significados gramaticales típicos de un cuantificador. Así, los casos como (5), se explican mejor postulando que todo tiene una función adverbial todo $\mathrm{FADV}_{\mathrm{F}}$, que consiste en modificar el grado de las propiedades expresadas por adjetivos y participios (RAE/ASALE, 2009). En 
los ejemplos mencionados, toda modifica una propiedad asignando un valor de totalidad. En inglés sucede algo muy similar. De acuerdo con Brinton y Traugott (2005), este valor adverbial — que también se ha denominado "intensificador" - , agrupa tanto el significado de totalidad como el grado de una propiedad. En inglés, all puede modificar adjetivos, participios y verbos en gerundio, lo cual se ilustra en los siguientes ejemplos de Waksler (2001, como se cita en Buchstaller \& Traugott, 2006):

(6) a. So I opened it, and my mother was all mad.

b. She was all screaming about the guy.

Por otra parte, es preciso mencionar que cuando precede a un adjetivo o un participio, todo no siempre tiene una función adverbial. Consideremos los siguientes ejemplos:

(7) a. Las señoras estaban todas angustiadas

\section{b. Las mesas estaban todas ocupadas}

En (7a), todas manifiesta la función adverbial anteriormente señalada. En (7b), por el contrario, todas es un cuantificador que aparece separado de su grupo sintáctico, de modo que la forma equivale a la expresión Todas las mesas estaban ocupadas. Esta capacidad del cuantificador de "moverse" a una posición posverbal ha sido denominada en la literatura como "cuantificación flotante" $\left(\operatorname{todo}_{\mathrm{FCF}}\right)$. En su uso adverbial, en cambio, no es posible "mover" todo a una posición prenominal (cf. *Toda Minnie se quedó temblorosa). Nótese que, en muchos casos, la posición posverbal de este elemento genera una posible ambigüedad entre lecturas de todo $_{\mathrm{FCF}}$ y lecturas de todo $\mathrm{FADV}_{\mathrm{FAD}}$ (Rickford, Wasow, Zwicky \& Buchstaller, 2007):

(8) Las calles se veían todas vacías

a. Todas las calles se veían vacías ( todo $_{\mathrm{FCF}}$ )

b. Las calles se veían completamente vacías (todo $\mathrm{FADV}_{\mathrm{FA}}$ )

Por último, existe un uso adicional de todo que se evidencia cuando aparece modificando un nombre, sin que se trate de una relación típica de cuantificación nominal. Se trata de un uso con función atributiva $\left(\right.$ todo $_{\text {FATR }}$ ) como en los ejemplos siguientes (tomados de RAE/ASALE, 2009): 
(9) a. María era toda corazón.

b. Eres todo nervios.

Hasta este punto, la presentación resume el panorama teórico en torno a las funciones semánticas de todo, como se muestra en la Tabla 1:

Tabla 1.

Funciones de "todo" en español.

\begin{tabular}{lll}
\hline Función & Nombre & Ejemplo \\
\hline Cuantificación & todo $_{\mathrm{FC}}$ & Todas las casas son blancas. \\
Cuantificación flotante & todo $_{\mathrm{FCF}}$ & Las casas son todas blancas. \\
Pronominal & todo $_{\mathrm{FPRO}}$ & Todas son blancas. \\
Adverbial & todo $_{\mathrm{FADV}}$ & La casa está toda desordenada. \\
Atributiva & todo $_{\mathrm{FATR}}$ & La casa es toda paz. \\
\hline
\end{tabular}

Fuente: Elaboración propia.

Al considerar en conjunto las funciones de todo, surge la pregunta de cuál es la relación entre ellas. En principio, se puede hipotetizar que la función cuantificacional es la más básica y a partir de ella se generan las otras funciones. Por ejemplo, debido a la fusión de los significados de grado y totalidad que se da en todo ${ }_{\mathrm{FADV}}$, es posible suponer que las funciones todo $\mathrm{FC}_{\mathrm{FC}} \mathrm{y}$ todo $\mathrm{FADV}_{\mathrm{F}}$ están estrechamente relacionadas en términos de la idea de cantidad. El problema principal de esta suposición está en la sintaxis. Cuando todo ${ }_{\mathrm{FADV}}$ precede a un predicado adjetival, su significado puede ser o no adverbial ('completamente'), pero su posición está determinada solo parcialmente por la naturaleza del adjetivo, como en el caso de (5b). En el Oxford English Dictionary, de acuerdo con Buchstaller y Traugott (2006), se hipotetiza que all con función adverbial (todo ${ }_{\mathrm{FADV}}$ ) proviene de all cuantificacional flotante $\left(\right.$ todo $\left._{\mathrm{FCF}}\right)$, pero esta hipótesis no está comprobada debido a que ambas funciones están presentes en el inglés antiguo, de modo que tanto en inglés como en español, esto se considera un asunto inconcluso (RAE/ASALE, 2009; Rickford et al., 2007). Sin embargo, la ambigüedad ejemplificada en (8) podría sugerir que el origen de todo ${ }_{\mathrm{FADV}}$ tiene que ver con la posición de todo ${ }_{\mathrm{FCF}}$. De hecho, Buchstaller y Traugott (2006) optan por apoyar la hipótesis de que la función de cuantificación y la 
adverbial como totalidad son tan cercanas que es plausible que la primera haya dado lugar a la segunda por efecto de reanálisis. En nuestro contexto, hay evidencia de que las funciones todo $\mathrm{FCF}_{\mathrm{FC}}(10), \operatorname{todo}_{\mathrm{FADV}}(11)$ $y$ todo $_{\text {FATR }}(12)$ estaban presentes en el español medieval (ejemplos tomados del CORDE):

(10) E aquellos Caldeos que los y metieron fueron todos quemados de la flama del fuego (c1200, La fazienda de Ultra Mar, Almerich, España).

(11) \& con vna cobertura toda Rota \& torpe ella toda ensangrentada de la sangre de sus hijos con dos pequeños sierbos aconpañada la mando hechar fuera de la çibdad (1402, Caída de príncipes, Pedro López de Ayala, España).

(12) E arribaron en la isla de Locaçia, que agora se llama Chipre, que era toda despoblada por cosarios e era toda montes. (1471-1476, Istoria de las bienandanzas e fortunas, Lope García de Salazar, España).

Dada la similitud de las propiedades gramaticales de todo y all, y teniendo en cuenta que se registran apariciones con dichas propiedades en la etapa incipiente del español, es posible plantear el argumento respecto del vínculo entre la función de cuantificación flotante y las funciones adverbial y atributiva como una inferencia a la mejor explicación. En todo caso, es claro que existen varias funciones semánticas relacionadas en términos de una noción general de cantidad y que se han documentado para las contrapartes de todo en otras lenguas como el inglés y el neerlandés (Buchstaller, 2014; Foolen, 2008).

\section{3. ¿Un cuantificador como introductor de discurso? El caso de all citativo}

Las funciones semánticas de "todo" mencionadas en la Tabla 1 coinciden con las que presenta el inglés all. Sin embargo, es posible identificar una función adicional de este elemento gramatical que los hablantes actualizan con el fin de introducir citas o DD. La presencia de esta función ha sido ampliamente documentada en inglés (Buchstaller, 2014; Buchstaller \&Traugott, 2006; Buchstaller, Rickford, Traugott, Wasow \& Zwicky, 2010; Rickford et al., 2007; Waksler, 2001). Al respecto, son ilustrativos los siguientes ejemplos tomados de Waksler (2001): 
(13) a. So I'm just ALL, "Can you just tell me how much our bill was?"

b. I said something funny, and he's ALL, "Write that down!"

c. Look the dog's ALL, "Wooooo."

d. I'm speeding down the hill, and I don't know how to stop, and I'm ALL [flails arms with terrified expression].

De acuerdo con esta última autora, expresiones como las de (13) dan cuenta de una distribución sintáctica y semántica del inglés all que difiere de aquella del cuantificador universal. No obstante, su uso como introductor de discurso se puede entender en términos de aquello que el hablante representa dentro de las citas. En (13a), el hablante introduce su propio discurso como DD, mientras que en (13b) reporta el discurso de alguien más. El caso de (13c) muestra que all introduce un discurso construido o ficticio en lugar de uno real. Finalmente, (13d) es usado para introducir comportamiento no verbal o citas gestuales. Para Waksler (2001), lo que representan estas citas - ya sean propias o ajenas, reales o construidas, verbales o no verbales-, es una caracterización total de un individuo en términos de ciertas propiedades relevantes. El adjetivo "total" es, justamente, el centro del argumento, puesto que la elección del cuantificador que denota totalidad para introducir DD se basa en su capacidad de hacer referencia a todos los elementos de un conjunto de propiedades que son atribuidas a la entidad de la cual se habla (es decir, de quien se reporta el discurso) en cada caso.

El uso de all como un introductor de DD se ha asociado con modos relativamente nuevos de habla y especialmente con interacciones informales en la modalidad oral. Mientras que el uso del elemento all como intensificador se puede rastrear hasta el inglés antiguo (Buchstaller \& Traugott, 2006) su uso citativo apareció en la década de 1980 en California y tuvo su punto más alto entre 2000 y 2004, aunque luego parecía haber caído en declive (Buchstaller et al., 2010; Rickford et al. 2007).

De acuerdo con Buchstaller y Traugott (2006), la función citativa del inglés like opera en una "escala de exactitud", de modo que la cita introducida por este marcador no necesariamente es fiel al discurso original que se reproduce. Lo mismo parece suceder con all, que sirve 
para señalar que "los hablantes están haciendo el mejor esfuerzo para reproducir la cita con tanta exactitud como es requerido por el propósito de la citación" (p. 361). Esto, señalan las autoras, es una hipótesis referida al inglés moderno, por lo que necesitaría algún tipo de comprobación o refutación.

En este punto, y teniendo en cuenta que todo tiene las mismas funciones semánticas que en inglés, surge la pregunta de si es posible identificar en español una función citativa todo ${ }_{\mathrm{FCIT}}$ como contraparte del llamado "quotative ALL". En lo que resta del trabajo, se propone que la respuesta a esta pregunta es afirmativa. Dadas las similitudes entre ambas lenguas, la estructura de las expresiones citativas con todo $\mathrm{FCIT}_{\mathrm{F}}$ sería, en principio, como la representada en (14). Justamente este es el tipo de estructura que tiene el ejemplo (15), extraído de Twitter sin modificaciones (aparte del énfasis):

(14) Sujeto (SER) todo: "cita"

(15) Amigos quería contarles lo duro que es correr en pasto, yo toda "pfff son $5 k$ que [sic] puede salir mal" (Twitter)

El caso de (15) no es aislado. Como se muestra a continuación, es posible identificar usos de todo ${ }_{\mathrm{FCIT}}$ como una manifestación concreta de que esta función citativa efectivamente tiene lugar al menos en el ciberlenguaje o lenguaje de internet, y que probablemente es un reflejo del mismo uso en el habla informal de las nuevas generaciones.

\section{Metodología}

Específicamente en torno al inglés, Buchstaller y Traugott (2006), Buchstaller et al. (2010) y Rickford et al. (2007) describieron la distribución estadística de all en contextos de modificación adverbial y citación. A diferencia de estos estudios de corte cuantitativo, la presente discusión apunta a describir la unidad todo ${ }_{\mathrm{FCIT}}$ que no ha sido tratada en español; por tanto, supone una aproximación cualitativa. Si bien es necesario un estudio cuantitativo basado en corpus para determinar el estado de todo y sus usos alternativos en nuestra lengua, el alcance del presente trabajo excluyó dicho objetivo. Los datos recogidos se vincularon más con un interés por mostrar que la expresión lingüística estudiada, en efecto, se registra en un tipo de modalidad lingüística, haciendo énfasis en las características semántico-pragmáticas de esta 
unidad. De este modo, es posible allanar el terreno para trabajos explicativos y estadísticos posteriores acerca del paradigma discutido, y en distintas variedades del español.

\subsection{Caracterización del corpus}

Los datos incluidos en este trabajo provienen de un corpus recolectado específicamente para este estudio. Los ejemplos fueron tomados de la red social Twitter. Las búsquedas realizadas corresponden a contextos en los que todo precede a algún tipo de enunciado expresado como citación, en relación con otros elementos que introducen valores epistémicos, evidenciales o subjetivos, o en casos en los que la mejor interpretación que se puede identificar corresponde a alguna de estas opciones.

\subsection{Procedimiento de recolección}

El procedimiento utilizado para la recolección de los datos tomados de Twitter involucra el programa RStudio (RStudio Team, 2020) y los paquetes que sirven para recolectar datos de la red social Twitter. Básicamente, se trata de un código que contiene la información para acceder a una cuenta personal de Twitter y un entorno gráfico para hacer búsquedas en la interfaz de programación de aplicaciones o "API", como se conoce en inglés. Los casos obtenidos de las búsquedas se pueden visualizar en el software de RStudio, además de poder ser guardados en un archivo de texto para su análisis posterior.

En particular, las búsquedas se realizaron usando el paradigma de pronombres del español. De acuerdo con la estructura de los ejemplos en inglés mencionados antes, lo usual es ver a all sucediendo un pronombre personal y la forma correspondiente del verbo to be (v. gr. She's all + "cita"). En el caso del español, no disponemos de argumentos para confirmar ni refutar la idea de que el verbo auxiliar deba ser parte de la construcción citativa. Sin embargo, vale la pena rescatar la presencia del pronombre, ya que al incluirlo dentro de la secuencia de búsqueda se puede reducir la gran cantidad de datos que se obtendría si solo se buscara todo (porque aparecerían muchos casos de las otras funciones de esta unidad). De este modo, y siguiendo la pista que deja el inglés, el procedimiento utilizado en este estudio consistió en llevar a cabo búsquedas de tuits con dos tipos de construcciones. La primera fue la 
forma "pronombre + todo/a/os/as", tratando de identificar la función citativa. La segunda fue "todo/a/os/as" seguido de expresiones oracionales que correspondieran a enunciados comunicados como DD. En algunos casos, el DD era evidente por la presencia de comillas, pero en otros no, de modo que se tuvieron en cuenta otras indicaciones (ver sección 5).

Además de la estructura de las búsquedas, el código de R usado incluyó los siguientes factores:

- Se hizo especificación de lengua ("es" para español), con el fin de evitar resultados en portugués (que también usa las mismas formas todo/a/os/as).

- Se usó un filtro para excluir retuits y así evitar las repeticiones del mismo texto.

- No se especificó la geolocalización, de modo que no es posible establecer si los resultados provienen de un país u otro. Esto puede entenderse como una limitación si la orientación es sociolingüística, o como una ventaja si se trata de establecer que la función citativa se presenta en el español en general (aunque, obviamente, no es una prueba en sí misma). En cualquier caso, las búsquedas se realizaron desde un computador conectado a internet en Santiago de Chile.

- Cada búsqueda se realizó una sola vez en el mismo día. Esto es importante porque la interfaz tiene una limitación respecto del número de tuits que puede buscar, de modo que esto afecta la posibilidad de encontrar tuits anteriores a una determinada fecha si se supera el máximo permitido hacia atrás en el tiempo. Aunque esta situación puede superarse con un parámetro que especifica la búsqueda entre dos fechas determinadas, es solo una solución parcial. En cualquier caso, esto no constituyó un obstáculo insalvable para el estudio.

En cuanto a los tuits como fuente de información lingüística, es preciso hacer algunas aclaraciones. En contraste con un motor de búsqueda que, por ejemplo, entrega una cantidad excesiva de información desplegada en miles de millones de páginas, Twitter tiene la ventaja de que cada tuit es lo suficientemente breve como para expresar una idea, construirla mediante los recursos de la lengua, y almacenarla en 
un espacio al que se puede acceder por medio de procedimientos de búsqueda de texto. Por desgracia, tanto el motor como la red social imponen limitaciones que es posible omitir. En primer lugar, Twitter no es un corpus en sí mismo, sino que tiene que ser procesado para llegar a convertirse en la fuente de un corpus creado durante el proceso de investigación y para fines específicos. También es un conjunto de datos "no limpios", en el sentido de que las unidades lingüísticas no están etiquetadas, ni separadas por criterios que sirvan para evitar repeticiones o casos que puedan quedar dentro del alcance de la búsqueda, pero que no sean casos relevantes.

Por otra parte, sería ingenuo pensar que los usuarios de las redes sociales respetan sistemáticamente la ortografía del español. Teniendo en cuenta esta realidad del lenguaje de internet, los tuits se reproducen exactamente como fueron recogidos en las búsquedas. De hecho, existen casos en los que la escritura no convencional o alejada de la norma es precisamente la indicación de que se está usando la unidad con la función que se investiga. En el apartado de resultados se ejemplifica de manera concreta esta situación.

A pesar de los inconvenientes mencionados, resulta razonable considerar a Twitter como una fuente interesante de fenómenos lingüísticos. Específicamente relevante para el presente trabajo es el hecho de que muchos tuits parecen reflejar más un uso informal que uno formal, incluso asemejándose a transcripciones de la modalidad oral de la lengua. En cualquier caso, lo importante es que los usos

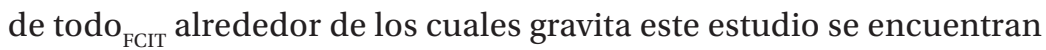
efectivamente registrados en tuits (y probablemente en otros espacios de redes sociales, foros, comentarios online, etc.). De este modo, la aparición en Twitter de las formas mencionadas respalda la elección de esta red como fuente de datos y justifica su utilización como origen de investigaciones lingüísticas.

Finalmente, es necesario reconocer que el uso de datos provenientes de redes sociales supone un conjunto de consideraciones éticas particulares para la investigación lingüística. Según Koene y Adolphs (2015) es preciso recordar que, aunque los usuarios saben que sus tuits son públicos (incluso si no leen los términos de la red al registrarse), no necesariamente estarían dispuestos a que esos tuits fueran analizados. $\mathrm{Al}$ respecto, estos autores sostienen que es necesario ser transparente 
respecto de los objetivos y los métodos de la investigación, además de obtener un consentimiento informado o darles a los usuarios la opción de ser excluidos. En la práctica, esto parece ser un problema de dimensiones incalculables si los datos provienen de millones de usuarios. Una manera básica de lidiar con esto es omitir el nombre de usuario que corresponde a cada tuit en el reporte de los datos (v. gr., una referencia genérica tipo @USUARIO), pero este intento de anonimizar no es suficiente, ya que el texto del tuit puede ser rastreado hasta la fuente mediante una búsqueda simple en internet. En el presente estudio, si bien se toma en cuenta la advertencia de los autores mencionados, se parte del supuesto de que los tuits de cuentas no restringidas son datos públicos, y son susceptibles de análisis con fines académicos, siempre que se traten con la responsabilidad ética usual que debe caracterizar la investigación científica del lenguaje.

\section{Resultados}

A continuación, se presentan algunos de los casos sic erat scriptum obtenidos en las búsquedas de tuits que ejemplifican el uso de todo ${ }_{\mathrm{FCIT}}$ :

16) Amigos quería contarles lo duro que es correr en pasto, yo toda "pfff son $5 k$ que puede salir mal"

17) Me preguntaron como 5 veces QUE VA A HACER HOY y yo toda NADA PORQUE NADIE ME SACA

18) Hasta Jimin nos dice que hagamos amigos y nosotros todos "en mi cueva estoy mejor" $x d$

19) Es un amor-odio el que se tienen ese par XDD ya lo sabíamos pero verlo así es más chistoso. Ella toda "ya déjame estúpido humano" todo el tiempo. La amo. Es mi meme fav después de Jjun XD

20) Jajaja jaja total. El todo tímido y ella toda Un placer Chau.

21) Yo también JAJSJAJSJAJA yo toda como ush que desocupado el que vaya hasta el centro comercial a decir eso Jajajakajs que fracaso

22) Mi cuñada quien está embarazada y mi mamá hablándole a la panza todas DÓNDE TA BEBÉ? NIIÑINIINI y yo toda NO LE HABLEN COMO MONGÓLICAS QUE SE TIRAN A LA CRÍAAAA, BOBASSSS 
23) JAJAJSJSJAJSJAJA SIIII y él todo, TRANQUILA, VIENEN CONMIGO JAJSJAJSKAJA y la señora de seguridad ahí toda ;-; no les creo $x d$ de paso los que c quedan encerrados JAJSJAJAJA

24) Me robaste el tuit iba a decir que Gumi estaria toda "ya ven los coreanos somos buena onda y bien geniales..... ya les hice el paro ahora ustedes en la casa de las waifus"

En términos estructurales, los casos (16), (18), (19) y (24) muestran claramente la función citativa de todo al preceder una expresión introducida con comillas. Los ejemplos (17), (20), (22) y (23) usan mayúsculas (iniciales o fijas) para marcar la distinción entre la citación y la expresión que la incluye. El tuit reproducido en (21) es el único que no tiene una marca ortográfica que sirva para identificar la citación. Sin embargo, cuenta como un ejemplo positivo de función citativa porque introduce una interjección (ush) y una exclamación (que [sic] desocupado el que vaya hasta el centro comercial). Ambas expresiones son típicas de casos de discurso referido que sucede a un pronombre y un marcador citativo, además de que su contenido en el contexto del tuit sugiere que se trata de una opinión expresada como una oración en forma de citación.

Como se había mencionado en la sección de antecedentes teóricos, estas expresiones no necesariamente corresponden a DD proveniente de un interlocutor o una fuente específica, sino que pueden ser pensamientos propios o discurso inventado expresado como discurso citado. En ocasiones, es posible que resulte difícil determinar si la expresión citada es un pensamiento propio del hablante o la reconstrucción de lo que diría la persona que aparentemente se cita. De cualquier forma, está claro que los diversos casos tienen cabida dentro del marco descriptivo propuesto.

\section{Discusión}

Teniendo en cuenta los datos obtenidos, surgen varias preguntas relacionadas con el uso de todo como elemento introductor de DD. En primer lugar, es posible preguntarse por el origen de este uso. Con el fin de aproximarse a una respuesta probable, el primer paso por dar es la comparación con lo que ha sucedido en otras lenguas. Como se mencionó antes, Buchstaller y Traugott (2006) sugieren que la función 
adverbial de all es el resultado de un proceso de reanálisis del alcance del operador all con función cuantificacional en posición flotante. El efecto de este cambio, añaden las autoras, se manifiesta en que el uso de all como adverbio se enfoca en el resultado que afecta a la entidad caracterizada y no en la totalidad espacial, temporal, etc., del evento. En este sentido, la función adverbial de all responde a los intereses de la conversación, de modo que su significado cuantificacional ha sufrido un proceso de gramaticalización ${ }^{3}$.

En el caso del español, es muy fácil obtener ejemplos de que todo también se utiliza en contextos discursivos similares a los ejemplificados en inglés:

(25) Ya es verano y yo toda despeinada (Twitter)

(26) Yo toda idiota ilusionandome con que la gente es mi amiga y se preocupan por mi, y luego me doy cuenta que no les importa (Twitter)

(27) Yo toda: Mi linaje y casta terminan conmigo (Twitter)

En los ejemplos (25) y (26), toda carece del valor cuantitativo que se le asigna típicamente en su posición como determinante, es decir, no es un operador que sirva para referirse a la totalidad de los elementos del conjunto expresado por el nombre al que determina. En el caso de (27), está claro que toda no reemplaza un nombre (función pronominal), no tiene alcance sobre un conjunto de elementos (función cuantificacional), no modifica el grado de un adjetivo o un participio (función adverbial), ni modifica un nominal con relación a una cualidad asociada a este (función atributiva). Como su contraparte inglesa, toda en (27) introduce una oración (función citativa). Existen al menos dos explicaciones posibles para este fenómeno. La primera es la que sigue el camino del cambio lingüístico a través de diversos procesos de gramaticalización. La segunda es que se trata de una copia del inglés, quizás por contacto lingüístico. No se cuenta con propuestas que apoyen definitivamente una u otra explicación, de modo que se pueden revisar los argumentos sobre los que descansan con el fin de establecer (quizás por abducción)

Buchstaller y Traugott (2006) no describen su hipótesis en estos términos, pero la interpretación de lo que ellas sugieren se puede parafrasear de la manera propuesta. Lo importante es tener en cuenta la idea del proceso de reanálisis que sufrió all en su función cuantificacional. 
cuál de ellas es la más adecuada. A continuación, se aborda brevemente a la segunda explicación y luego se profundizará en la primera.

\subsection{Transferencia lingüística}

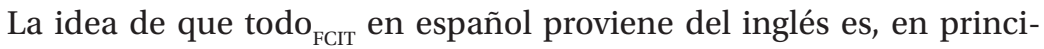
pio, verosímil. En primer lugar, all y todo en estas lenguas presentan las mismas funciones y estructuras discursivas en los casos relevantes. Por otra parte, no solo existen contextos específicos de contacto lingüístico entre ambas lenguas (por ejemplo, en EE.UU.), sino que la influencia cultural del inglés es muy fuerte incluso en países donde no hay situaciones de bilingüismo ni contacto directo con hablantes nativos. Así, no es imposible que estructuras como "I was all + DD" pudieran ser copiadas y adaptadas al español. Sin embargo, existen varios aspectos que van en contra de esta aparentemente simple explicación. El primero es que la versión inglesa solo admite estructuras $B E$ all, mientras que en español la variedad es mayor, pudiendo prescindir del verbo SER. El segundo aspecto es que la influencia del inglés, si bien es grande, no es un factor que garantice con certeza la ocurrencia de la copia. Al respecto es interesante señalar el caso del marcador discursivo como en el trabajo de Said-Mohand (2008). Sus observaciones en hablantes bilingües incluyen registros de estructuras en las que como introduce discurso directo. Sin embargo, este autor menciona que no hay evidencia concluyente para afirmar que la función de introducir DD de como sea una transferencia proveniente del inglés “like + DD"4. Del mismo modo, no es posible afirmar que el todo ${ }_{\mathrm{FCIT}}$ de este estudio es una transferencia de all hasta que no se cuente con estudios sociolingüísticos a gran escala. Los datos obtenidos aquí no se limitan a una variedad específica del español, así que en sí mismos no pueden confirmar la hipótesis de la transferencia lingüística.

En una línea similar, Cheshire y Secova (2018) sostienen que el paradigma de nuevos citativos en francés (que incluye expresiones como

\footnotetext{
$4 \quad$ Al respecto, trabajos como los de la investigadora Wiltrud Mihatsch (sugeridos por un evaluador) también hacen referencia al como en su valor de marcador citativo. Mihatsch (2010) comenta el trabajo de Said-Mohand (2008) acerca de esta unidad en hablantes de español e inglés en EE.UU., pero afirma que este uso de introducción de citas no existe en variedades sin contacto, algo con lo que no estoy de acuerdo. En cualquier caso, la relación entre las funciones pragmáticas discursivas de like y como es un tema interesante como contraparte de la relación entre all y todo.
} 
genre y etre la) ha sufrido transformaciones recientes debido a las innovaciones lingüísticas que aportan principalmente las generaciones jóvenes. Su estudio concluye que los citativos de similitud (es decir, de características parecidas a las de $B E$ like) no son copias del inglés, pero sí han entrado al sistema citativo del francés del mismo modo en el que like entró al sistema inglés, es decir, apareciendo en diversos contextos sintácticos y cumpliendo nuevas funciones pragmáticas. Esta idea está en consonancia con la explicación alternativa, que se discute a continuación.

\subsection{Gramaticalización como expansión y analogía}

La segunda explicación mencionada acerca del origen de todo ${ }_{\mathrm{FCIT}}$ es la que tiene que ver con procesos de cambio lingüístico propios del cuantificador en español. Una generalización simple acerca de las funciones semánticas de todo es que sirven al propósito de introducir cierta información en relación con el hablante o con alguien/algo distinto. En algunos casos se trata de información cuantitativa, en otros de información cualitativa. En cuanto a la función pragmática todo ${ }_{\mathrm{FCIT}}$, $\mathrm{se}$ puede hipotetizar que, para llegar a su estado de unidad citativa, todo ha pasado por al menos dos etapas de cambio lingüístico. En la primera etapa, todo pudo haber perdido su significado de cantidad específica para dar lugar a un significado de atribución total de una propiedad. En la segunda etapa, mucho más reciente, todo ha adoptado un valor discursivo que permite introducir formas de citación, comparable con el de "decir" en contextos de discurso directo. El centro de la discusión tiene que ver con la manera en que estas dos etapas de significado están relacionadas.

Como se mencionó anteriormente, las descripciones semánticas de todo no hacen referencia a los usos discursivos en contextos de citación. En este sentido, el paradigma de los citativos en español necesita revisión, de acuerdo con los fenómenos recientes tanto en nuestra lengua como en otras de las familias romance y germánica. Esto va mucho más allá del alcance del presente estudio, pero un primer paso en esa dirección es examinar la función citativa en términos de su origen a partir de procesos de cambio lingüístico. Para ello, es preciso contextualizar una posición teórica respecto de la gramaticalización. Una definición operativa de esta noción, que muchos estudios suponen, es que se trata de un proceso de cambio que afecta a 
una unidad lingüística, de modo que su significado léxico (o gramatical) se vuelve (más) gramatical. De acuerdo con Hopper y Traugott (2003), el término especializado "gramaticalización" tiene dos significados: uno que se refiere a un conjunto de fenómenos de cambio lingüístico y otro que alude al marco de investigación que estudia dichos fenómenos. La gramaticalización es un tema tan profusamente discutido que no está exento de controversias (Applebaum, 2014; Lehmann, 2015; Traugott, 2010). Para efectos del presente estudio, se tiene en cuenta el contraste que propone Traugott (2010) entre gramaticalización entendida de manera tradicional como una pérdida o reducción de propiedades de un elemento lingüístico y la gramaticalización entendida como el resultado de procesos expansión. Esta última es adoptada por Himmelmann (2004) para defender que, si bien se ha entendido que los elementos léxicos o gramaticales pueden perder funciones semánticas y adquirir significados más específicos y abstractos, es más adecuado considerar que la gramaticalización es un proceso por el que un elemento no aislado sino en contexto (una construcción) puede adquirir propiedades nuevas.

Según Himmelmann (2004), en la perspectiva de la gramaticalización como proceso de expansión es posible distinguir entre expansión de clase, expansión sintáctica y expansión semántico-pragmática. La primera tiene que ver con la reorganización de los paradigmas, que en el uso de la lengua pueden adquirir o perder formas y configurar nuevas relaciones entre sus elementos. La segunda hace referencia al cambio que puede sufrir el contexto sintáctico en el que aparece una forma particular. La tercera expansión, la más importante según Himmelmann, permite que una construcción amplíe los posibles contextos en los que ocurre, aumentando así sus opciones de transmitir nuevos significados y, con ellos, nuevos usos. Como se verá respecto de los datos obtenidos acerca de la función citativa de todo, esta aproximación a la gramaticalización como expansión resulta adecuada para describir las posibles etapas que permitieron el uso actual de todo como introductor de discurso.

Uno de los puntos de partida de este trabajo fue que las funciones de todo y sus variaciones formales están relacionadas con un conjunto de cambios lingüísticos específicos que han tenido lugar en español y en varias lenguas emparentadas. Para efectos de la discusión, es posible 
sostener que las funciones de todo se relacionan entre sí de varias maneras. La función cuantificacional constituye el valor semántico más básico de esta forma lingüística, es decir, la dimensión semántica predeterminada del paradigma de todo. La función pronominal puede obtenerse por procesos de elisión y anáfora, que son transparentes en términos de la gramática del español (Todas las casas eran blancas $>$ Todas eran blancas). En cuanto a las funciones adverbial y atributiva, y de acuerdo con la bibliografía citada, parece razonable suponer que se originaron a partir del fenómeno de la cuantificación flotante (lo que, de nuevo, tendría que comprobarse con datos históricos). Estas dos funciones son más complejas que las expresadas por todo cuantificacional, dado que codifican al menos dos valores: uno de cantidad menos específico que el de todofc (es decir, no equivalente a 'cada uno de los elementos del conjunto denotado por el nombre', sino equivalente a 'completamente'), y una relación meronímica (sinécdoque) en la que se toma el todo por la parte. Por ejemplo, consideremos los siguientes casos:

\section{(28) María está toda distraída}

\section{(29) María es todo corazón}

En (28) se está haciendo referencia a que María está completamente distraída, cuando en realidad solo la dimensión mental de María es la que puede distraerse. En (29), se usa de nuevo el recurso de la meronimia para dar a entender que la personalidad de María se caracteriza por los atributos positivos asociados figurativamente con el corazón. Así, se tiene que las funciones reconocidas de todo son el resultado de factores sintácticos y semánticos de su historia dentro del sistema de la lengua. La Tabla 2, presentada más abajo, sirve como guía para resumir lo dicho y explorar la cuestión final relativa a todofCIT:

Tabla 2 .

Funciones y gramaticalización de "todo" en español.

\begin{tabular}{|c|c|c|c|}
\hline \multicolumn{3}{|c|}{ FUNCIÓN } & PROCESO \\
\hline \multirow{4}{*}{ todofC } & $>$ & todo $_{\text {FPRO }}$ & elipsis/anáfora \\
\hline & $>$ & todo $_{\text {FADV }}$ & \multirow{2}{*}{ cuantificación flotante y meronimia } \\
\hline & $>$ & todoFATR & \\
\hline & $>$ & todo ${ }_{\text {FCIT }}$ & $?$ \\
\hline
\end{tabular}

Fuente: Elaboración propia. 
En la última fila de la tabla aparece la función citativa, que es el fenómeno central abordado en la presente discusión. Si los procesos de cambio lingüístico que afectaron a todo en español son, como se ha sostenido, similares a los que sufrió el inglés all, podríamos suponer que las distintas etapas del uso de este elemento corresponderían a situaciones en las que la función primigenia se reanaliza como otra, la que a su vez sirve de base para otro proceso de reanálisis. Esta cadena de cambios se puede ejemplificar con un orden como el siguiente:

(E1) Todos mis amigos son divertidos

(E2) Mis amigos son todos divertidos

(E3) Mis amigos (son) todos divertidos

(E4) Mis amigos todos “'Somos divertidos!”

En el caso del primer estadio (E1), todo aparece en su función cuantificacional original. La forma en la segunda etapa (E2) ilustra la operación de cuantificación flotante. La siguiente etapa, E3, puede entenderse como el desplazamiento que sufre la función cuantificacional de todo hacia una función adverbial, que en otros contextos puede ser atributiva. Nótese que las construcciones en E2 y E3 son idénticas, pero su análisis estructural no es el mismo: en E2 tenemos todos como un cuantificador desplazado o flotante, mientras que en E3 tenemos un adverbio que modifica al adjetivo dentro del sintagma verbal (con el significado de "completamente divertidos"). El verbo en E3 aparece entre paréntesis debido a que la función adverbial se puede expresar sin este elemento, como lo muestran los abundantes casos de construcciones con estructura [pronombre + todo + adjetivo].

Finalmente, la situación mostrada en E4 intenta representar la función citativa, no reproduciendo un discurso previo proferido concretamente, sino como la expresión por parte de un hablante de una proposición que se manifiesta como una citación. La reconstrucción no pretende ser exhaustiva, ya que existen varios aspectos que no se reflejan en esta simplificación. Lo importante es tratar de establecer una posible relación entre un valor gramatical de cuantificación y un valor pragmático de citación. Siguiendo a Himmelmann (2004), y sin estudios diacrónicos disponibles a la fecha, una explicación provisio-

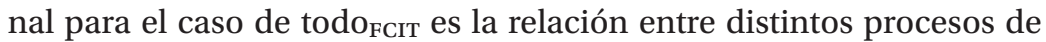
expansión. Por un lado, la expansión de clase le ha permitido al para- 
digma de los citativos incluir variantes nuevas, acomodando opciones que difieren de los elementos tradicionales (los verbos de cita y otras formas recientes). Por otra parte, la expansión de contexto sintáctico podría explicar el hecho de que la estructura cuantificacional se ha reorganizado a partir de los procesos de elipsis, anáfora y cuantificación flotante (como se explicó anteriormente). Por último, la expansión semántica involucra las posibilidades de significado derivadas de los procesos sintácticos (valores de cantidad menos específica, atribución de propiedades y meronimia), mientras que la expansión pragmática le ha permitido a todo figurar en contextos de citación que no tenía en estados anteriores de su evolución.

Un asunto por tratar es el de por qué el elemento todo no pierde la marcación de flexión, algo que es esperable en un proceso de gramaticalización. Es importante señalar que en el caso de la función todo fatr sí se pueden presentar casos de pérdida de la concordancia, como lo muestran los ejemplos de RAE/ASALE (2009): “María era todo corazón", “Eres todo nervios". La misma gramática señala que en el español de América se dan casos como "La muchacha iba todo asustada", en los que la falta de concordancia es consistente con la interpretación adverbial de todo. En este escenario es completamente natural preguntarse por qué todoFCIT mantiene la concordancia. Al respecto, existen varias opciones. En primer lugar, se podría simplemente decir que el todo ${ }_{\mathrm{FC}}$ no tiene que ver con la gramaticalización. Por ejemplo, Lehmann (2015) advierte que la distribución extendida de una forma no garantiza que se trate de un elemento gramaticalizado, porque también podría referirse a un caso de transferencia analógica ${ }^{5}$. Esto, sin embargo, no quiere decir que la decisión sea excluyente. Fischer (2011) señala que si bien la gramaticalización existe como proceso, solo se trata de una generalización del analista: lo que en realidad ocurre en la lengua es un cambio lingüístico que se puede explicar en términos de la analogía. Para el caso de este estudio, el hecho de que todo permite a los hablantes expresar una propiedad y un sentido de totalidad asociado a ella podría ser la fuente de similitud que ha dado lugar al uso de este elemento como introductor de DD, en la medida en que dicha propiedad puede

El autor señala que tratará con más detalle este asunto en una sección posterior del libro, pero esta sección no ha sido incluida en la versión que está disponible a la fecha. 
transmitirse con mayor expresividad si se presenta como una cita, ya sea propia o ajena, real o creada para los propósitos del intercambio comunicativo que tiene lugar.

\section{Conclusiones}

Lo dicho hasta el momento no es más que una propuesta exploratoria y está lejos de llenar los vacíos que existen respecto de los fenómenos recientes que relacionan unidades discursivas con procesos de gramaticalización. Se necesitan diversos tipos de estudios que complementen lo que se sabe acerca de los nuevos citativos y que ofrezcan explicaciones adecuadas a conjuntos de datos mucho más grandes. Por ejemplo, si se usan redes sociales como fuente de información lingüística, es preciso contar con investigaciones sociolingüísticas que manipulen variables como la geolocalización (tuits por país) y las fechas de aparición (Twitter se lanzó en 2006).

Por otra parte, una pregunta que queda sin responder es si este cambio lingüístico, que al parecer lleva un par de generaciones a lo sumo, está en declive o en aumento. Para el inglés, los datos de Buchstaller et al. (2010) sugieren que all no fue sino una "short-lived innovation" que cedió su espacio en el paradigma al citativo like después de un declive en su uso alrededor del año 2005. Dado que no se contó con datos estadísticos para el todo en español, no es posible establecer si sucede lo mismo que en inglés o si se va en la dirección contraria. Al respecto, la contribución de las nuevas formas de interacción social en internet es un factor clave, no solo por la disponibilidad de los datos y las herramientas computacionales que se han desarrollado para tratarlos, sino porque no se dispone de muchos corpus que tengan las características necesarias para estudiar este fenómeno.

Finalmente, es necesario explorar otro tipo de factores asociados al uso de todo como marcador citativo. Una cuestión muy importante es la que tiene que ver con la multimodalidad. Muchas de las apariciones de todo $\mathrm{FCIT}_{\mathrm{F}}$ en Twitter preceden elementos que representan un discurso en formatos de diversa naturaleza semiótica: emoticones, emojis, memes, videos, archivos GIF, etc. Por ejemplo, uno de los tuits recuperados en la búsqueda para este trabajo fue "y yo toda: 6)". Este emoji, que según las prácticas del lenguaje de internet puede interpretarse 
como representación de vergüenza o asombro, podría constituir una cita de lenguaje no verbal.

De acuerdo con lo propuesto anteriormente, la función citativa se deriva de las funciones adverbial y atributiva y su uso meronímico. Además, parece razonable aceptar que el uso de todo FCIT $_{\text {se }}$ adapta perfectamente a las necesidades de los hablantes de expresar con más efusividad sus estados mentales, de modo que la introducción de DD resulta útil para comunicar lo que de algún modo está indeterminado por la lengua (por ejemplo, un simple contraste entre "yo todo cansado" y "yo todo: 'estoy cansado"' puede marcar grandes diferencias de expresividad en términos de contenido, entonación, fuerza, etc.). Quizás el camino a seguir sea explorar la noción de subjetividad que proponen autores como Traugott y Dasher (2004), o tal vez deba ser otra alternativa funcionalista o cognitivista. En cualquier caso, lo importante es que se tenga en cuenta que resulta más adecuado en términos científicos explicar los datos considerando factores como las propiedades del sistema de la lengua, las relaciones de dependencia estructural y el contexto comunicativo en el que se dan estos fenómenos lingüísticos.

\section{Referencias bibliográficas}

Applebaum, I. (2014). Grammaticalization and explanation. En S. Hancil \& E. König (Eds.), Grammaticalization. Theory and data (pp. 41-52). Amsterdam: John Benjamins.

Brinton, L. J. \& Traugott, E. C. (2005). Lexicalization and grammaticalization all over again. En J. C. Salmons \& S. Dubenion-Smith (Eds.), Historical linguistics 2005: Selected papers from the 17th International Conference on Historical Linguistics, Madison, Wisconsin, 31 July - 5 August 2005 (pp. 3-19). Amsterdam: John Benjamins.

Buchstaller, I. (2014). Quotatives: New trends and sociolinguistic implications. Oxford: Wiley-Blackwell.

Buchstaller, I., Rickford, J. R., Traugott, E. C., Wasow, T., \& Zwicky, A. (2010). The sociolinguistics of a short-lived innovation: Tracing the development of quotative all across spoken and internet newsgroup data. Language Variation and Change, 22(2), 191-219. https://doi.org/10.1017/s0954394510000098 
Buchstaller, I. \& Traugott, E. C. (2006). The lady was all demonyak: Historical aspects of adverbial all. English Language and Linguistics, 10(2), 345-370. https://doi.org/10.1017/ s136067430600195x

Cameron, R. (1998). A variable syntax of speech, gesture, and sound effect: Direct quotations in Spanish. Language Variation and Change, 10(1), 43-83. https://doi.org/10.1017/ s0954394500001216

Cheshire, J. y Secova, M. (2018). The origins of new quotative expressions: the case of Paris French. Journal of French Language Studies 28, 209-234.

Demonte, V. y Fernández, O. (2013). El que citativo, otros que de la periferia izquierda oracional y la recomplementación. En D. Jakob y K. Plooj (Eds.), Autour de 'que' (pp. 47-69). Frankfurt: Peter Lang.

de Vaan, M. (2008). Etymological dictionary of Latin and the other Italic languages. Leiden: Brill.

Etxepare, R. (2008). On quotative constructions in Iberian Spanish. En R. Laury (Ed.) Crosslinguistic studies of clause combining: The multifunctionality of conjunctions. Amsterdam: John Benjamins.

Fischer, O. (2011). Grammaticalization as analogically driven change? En B. Heine \& H. Narrog (Eds.), The Oxford handbook of grammaticalization (pp. 31-42). Oxford: Oxford University Press.

Foolen, A. (2008). New quotative markers in spoken discourse. En B. Ahrenholz, U. Bredel, W. Klein, M. Rost-Roth, \& R. Skiba (Eds.), Empirische Forschung und Theoriebildung (pp. 117128). Frankfurt: Peter Lang.

Gallucci, M. J. (2017). Contribución al estudio del discurso referido en un corpus oral del español americano (Tesis doctoral). Universidad de Zaragoza, Zaragoza.

Garachana, M. (2015). Teoría de la gramaticalización. Estado de la cuestión. En T. Bastardín y M. Rivas (Eds.), Actas del IX Congreso Internacional de Historia de la Lengua Española, vol. 1 (pp. 331-360). Madrid: Iberoamericana-Vervuert.

Gutiérrez-Rexach, J. (2016). Cuantificación. En J. Gutiérrez-Rexach (Ed.), Enciclopedia de lingüistica hispánica, vol. 1 (pp. 506514). Londres: Routledge. 
Himmelmann, N. P. (2004). Lexicalization and grammaticalization: Opposite or orthogonal? En W. Bisang, N. Himmelmann, \& B. Wiemer (Eds.), What makes grammaticalization. A Look from its fringes and its components (pp. 9-40). Berlin: Mouton de Gruyter.

Hopper, P. J. \& Traugott, E. C. (2003). Grammaticalization. Cambridge: Cambridge University Press.

Jørgensen, A. (2012). Funciones del marcador pragmático "como" en el lenguaje juvenil español y chileno. En M. E. Placencia y C. García (Ed.), Pragmática y comunicación intercultural en el mundo hispanohablante, vol. 44 (pp. 209-231). Leiden: Brill.

Jørgensen, A. y Stenström, A. (2009). Dos marcadores pragmáticos contrastados en el lenguaje juvenil: el inglés like y el español como. Español Actual: Revista de Español Vivo, 92, 103-120.

Koene, A. \& Adolphs, S. (2015). Ethics considerations for corpus linguistic studies using internet resources. Recuperado de https:// casma.wp.horizon.ac.uk/wp-content/uploads/2015/04/ CL2015-CorpusLinguisticsEthics_KoeneAdolphs.pdf

Lehmann, C. (2015). Thoughts on grammaticalization (3rd edition). Berlin: Language Science Press.

Leonetti, M. (2007). Los cuantificadores. Madrid: Arco Libros.

Mihatsch, W. (2010). Sincronía y diacronía del aproximador como. Revista Internacional de Lingüística Iberoamericana, 8(2), 175201. Recuperado de https://www.jstor.org/stable/41678457

Palacios, I. (2013).Zero quotingin the speech of British and Spanish teenagers: A contrastive corpus-based study. Discourse Studies, 15(4), 439-462. https://doi.org/10.1177/1461445613482431

Panussis, C. y San Martín, A. (2017). Como (que) y sus funciones discursivas en el habla santiaguina: análisis pragmático y sociolingüístico. Revista de Lingüística Teórica y Aplicada, 55(2), 39-61. https://doi.org/10.4067/s0718-48832017000200039

Real Academia Española, CORDE (s/f). Corpus diacrónico del español (en línea). Recuperado de http://www.rae.es

Real Academia Española, RAE y Asociación de Academias de la Lengua Española, ASALE. (2009). Nueva gramática de la lengua española. Madrid: Espasa.

Rickford, J. R., Wasow, T., Zwicky, A., \& Buchstaller, I. (2007). Intensive and quotative all: Something old, something new. American 
Speech, 82(1), 3-31. https://doi.org/10.1215/00031283-2007001

RStudio Team (2020). RStudio: Integrated Development Environment for R. RStudio, PBC, Boston, MA. URL: http://www.rstudio. com/

Said-Mohand, A. (2008). Aproximación sociolingüística al uso del marcador del discurso como en el habla de jóvenes bilingües en la Florida. Revista Internacional de Lingüistica Iberoamericana 6(2), 71-93. Recuperado de https://www.jstor.org/stable/41678352

San Martín, A. y Guerrero, S. (2013). Una aproximación sociolingüística al empleo del discurso referido en el corpus PRESEEA de Santiago de Chile. Revista Signos 46(82), 258-282. https:// doi.org/10.4067/s0718-09342013000200005

Traugott, E. C. (2010). Grammaticalization. En S. Luraghi \& V. Bubenik (Eds.), The continuum companion to historical linguistics (pp. 271-285). Londres: Continuum.

Traugott, E. C. \& Dasher, R. B. (2004). Regularity in semantic change. Cambridge: Cambridge University Press.

Waksler, R. (2001). A new all in conversation. American Speech, 76(2), 128-138. https://doi.org/10.1215/00031283-76-2-128 